\title{
AMPA Receptors Exist in Tunable Mobile and Immobile Synaptic Fractions In Vivo
}

\author{
Haiwen Chen, ${ }^{1,2,3} \oplus^{-}$Richard H. Roth, ${ }^{3}$ Elena Lopez-Ortega, ${ }^{3}$ Han L. Tan, ${ }^{3}$ and ${ }^{\circledR}$ Richard L. Huganir ${ }^{3}$
}

https://doi.org/10.1523/ENEURO.0015-21.2021

${ }^{1}$ Division of Pediatric Neurology, Department of Neurology, Johns Hopkins University School of Medicine, MD 21205 ,

${ }^{2}$ Department of Pediatrics, Johns Hopkins University School of Medicine, MD 21205, and ${ }^{3}$ Solomon H. Snyder

Department of Neuroscience and Kavli Neuroscience Discovery Institute, Johns Hopkins University School of

Medicine, Baltimore, MD 21205

\begin{abstract}
AMPA receptor (AMPAR) mobility within synapses has been extensively studied in vitro. However, whether similar mobility properties apply to AMPARs in vivo has yet to be determined. Here, we use two-photon fluorescence recovery after photobleaching (FRAP) to study AMPAR mobility within individual dendritic spines in live animals using an overexpression vector. We demonstrate the existence of mobile and immobile fractions of AMPARs across multiple cortical regions and layers. Additionally, we find that AMPAR mobility can be altered in vivo in response to administration of corticosterone, a condition that mimics exposure to stress.
\end{abstract}

Key words: GluA1; plasticity; stress; synapses

\section{Significance Statement}

Our work provides novel insight into receptor mobility within intact brains of live mice using two-photon microscopy through cranial windows. In vivo assessment of protein mobility within mammalian neuronal synapses has thus far been limited. Here, within this system, we are able to confirm that there are both mobile and immobile AMPA receptor (AMPAR) fractions in vivo and that these fractions are similar across different cortical regions and layers. Additionally, we reveal that the proportion of mobile to immobile receptor fraction may be altered by administration of corticosterone, a condition that mimics stress response, suggesting AMPAR mobility is acutely modulated in vivo.

Received January 11, 2021; accepted April 12, 2021; First published April 27, 2021.

The authors declare no competing financial interests.

Author contributions: H.C. and R.L.H. designed research; H.C., R.H.R., E.L.-O., and H.L.T. performed research; H.C. analyzed data; H.C. and R.L.H. wrote the paper.

This work was supported by the National Institutes of Health Grant NS050274 (to the Johns Hopkins University Neuroscience Department Multiphoton Imaging Core) and by the National Institutes of Health Grant NS036715 (to R.L.H.).

Acknowledgements: We thank Michele Pucak and the Johns Hopkins University Neuroscience Department Multiphoton Imaging Core for use of the two-photon microscope.

Correspondence should be addressed to Richard L. Huganir at rhuganir@ jhmi.edu.

https://doi.org/10.1523/ENEURO.0015-21.2021

Copyright (c) 2021 Chen et al.

This is an open-access article distributed under the terms of the Creative Commons Attribution 4.0 International license, which permits unrestricted use, distribution and reproduction in any medium provided that the original work is properly attributed.

\section{Introduction}

Trafficking of AMPA receptors (AMPARs) modulates synaptic strength at excitatory synapses. This process underlies the plasticity of neurons that supports learning and memory (Huganir and Nicoll, 2013; Diering and Huganir, 2018). In postsynaptic spines, AMPARs laterally diffuse along the plasma membrane and may enter and exit synapses (Borgdorff and Choquet, 2002; Heine et al., 2008). Within synapses themselves, a combination of protein interactions and molecular crowding constrains and immobilizes a large fraction of AMPARs (Bats et al., 2007; Li et al., 2016). The clustered distributions of these synaptic AMPARs and their relative position to presynaptic vesicle release machinery further regulate the efficacy of synaptic transmission (MacGillavry et al., 2013; Nair et al., 2013; Tang et al., 2016).

AMPAR mobility has previously been assessed within individual synapses in vitro. One technique utilizes 
fluorescence recovery after photobleaching (FRAP) of fluorescently tagged AMPARs, whereby fluorescence recovery reflects exchange of bleached molecules in the spine with unbleached molecules elsewhere in the cell, representing a mobile fraction of molecules. Conversely, a lack of fluorescence recovery reflects the immobile protein fraction.

Within dissociated hippocampal cell cultures, FRAP of fluorescently tagged AMPAR subunit GluA1 revealed a $56 \%$ mobile fraction within dendritic spines (Sharma et al., 2006). Other such studies provided a range between $20 \%$ and 60\% (Ashby et al., 2006; Bats et al., 2007; Heine et al., 2008; Frischknecht et al., 2009; Martin et al., 2009; Arendt et al., 2010). Using super-resolution single molecule tracking, the mobile fraction of GluA1 was estimated to be $\sim 70 \%$, where half of these receptors exchanged between synaptic and extrasynaptic domains and the other half was mobile within the synapse (Heine et al., 2008). In contrast, within organotypic hippocampal cultures, Makino and Malinow (2009) found 103\% recovery of GluA1 with FRAP at basal states, suggesting that GluA1-containing AMPARs were entirely mobile. They proposed that this is because recombinant GluA1 remains within spine surface pools rather than being stably incorporated into synapses at basal states. They further observed a decrease in GluA1 FRAP recovery to $\sim 70 \%$ only with induction of chemical long-term potentiation (LTP), suggesting a portion of those receptors had been incorporated into the immobile synaptic fraction with stimulation, consistent with previous experiments on LTP in organotypic cultures (Hayashi et al., 2000; Kopec et al., 2006). In further contrast, within acute slices, FRAP of chemically labeled endogenous GluA1 was reported to recover only $\sim 10 \%$ fluorescence (Wakayama et al., 2017). Notably, there is overall significant disparity in the characterization of AMPAR dynamics between different experimental systems, in part because of differences between in vitro conditions. Which system most accurately reflects biology in vivo is unknown.

Therefore, we sought to assess AMPAR mobility at individual spines of cortical neurons in intact brains of live animals employing in vivo two-photon FRAP of fluorescently labeled AMPARs.

\section{Materials and Methods}

\section{Animals}

Experiments were performed using male and female adult (two to four months) wild-type (WT) C57BL/6N mice (Charles River). All animals were treated in accordance with the Johns Hopkins University Animal Care and Use Committee guidelines. Animals were kept on a 12/12 h light/dark cycle.

\section{In utero electroporation and cranial window implantation}

In utero electroporation and cranial window implantation were performed as previously described (Zhang et al., 2015; Roth et al., 2020; Tan et al., 2020). Briefly, in utero electroporation of SEP-GluA1, myc-GluA2, and
DsRed2 at a 4:2:1 ratio was performed on embryonic day (E)15 embryos from timed pregnant C57BL/6N mice in L2/3 of visual cortex or E13 embryos for L5 of either motor or visual cortices. Pups born after, both males and females, were implanted with a cranial window overlying respective transfected cortices at 8-12 weeks of age, and a custom-made metal head bar was attached to the skull to fixate the mouse for imaging.

\section{Two-photon FRAP imaging}

In vivo two-photon imaging was performed under isoflurane anesthesia $\left(0.5 \%\right.$ volume isoflurane/volume $\left.\mathrm{O}_{2}\right)$ using a Zeiss two-photon laser-scanning microscope with a $20 \times 1.0 \mathrm{NA}$ water immersion objective lens (Zeiss). SEP-GluA1 and DsRed2 were excited at $910 \mathrm{~nm}$ with a Ti: sapphire laser (Coherent) with $\sim 100-\mathrm{mW}$ power delivered to the back-aperture of the objective. Image stacks of 12$14 z$-steps were acquired at $512 \times 512$ pixels with pixel size of $0.21 \mu \mathrm{m}$ in $x$ and $y$ and $z$-steps of $1 \mu \mathrm{m}$ with pixel dwell times of $2 \mu \mathrm{s} /$ pixel. Baseline image stacks were acquired $5 \mathrm{~min}$ apart before bleaching and FRAP image stacks acquired immediately after photobleaching and at times indicated in the figures. Photobleaching of spines were achieved with repetitive xy scanning of select $10 \times 10$-pixel regions of interest (ROIs) at the center plane of the image stack at high illumination intensity at $910 \mathrm{~nm}$ for dwell times of 32-65 $\mu \mathrm{s} / \mathrm{pixel}$ with one to two iterations. For each experiment, $\sim 10-15$ spines were bleached at a time for total bleach times of 30-60 s. Spines targeted for photobleaching resulted in $\sim 50 \%$ reduction of fluorescence in SEP-GluA1 and $\sim 15 \%$ decrease in fluorescence in DsRed2 (Extended Data Fig. 1-1D).

\section{Corticosterone experiments}

First, baseline FRAP images were collected as described above. Then at least $1 \mathrm{~d}$ after acquisition of baseline images, either corticosterone (water soluble 2hydroxypropyl- $\beta$-cyclodextrin complex, Sigma-Aldrich, dissolved in $0.9 \%$ normal saline) at $5 \mathrm{mg} / \mathrm{kg}$ or the same volume of $0.9 \%$ normal saline was injected intraperitoneally. FRAP images were subsequently collected at 1,2 , and $3 \mathrm{~h}$ postinjection in three distinct regions. On a third day, at least $3 \mathrm{~d}$ following the previous injection, the injection of the other substance was performed followed by the same imaging protocol. This set of experiments included only mice with neurons electroporated in L2/3 of visual cortex. Only data from animals where all $3 \mathrm{~d}$ of data were able to be collected were included in the analysis. Because of the circadian nature of endogenous corticosterone release in mice, all experiments were performed within a 5-h time window between 1 and 6 P.M. to minimize the contribution of innate circadian corticosterone variation to the experimental readout.

\section{Image analysis}

Image processing and analysis were performed in ImageJ following export from Zen (Zeiss). Values measured in ImageJ were subsequently analyzed using custom 
scripts in MATLAB. All image processing was batched and performed blinded to group or condition, which were later reassigned on performing statistical analyses. Image $z$-stacks at all time points were maximally projected in the z-dimension. StackRegJ (Jay Unruh, Stowers Institute for Medical Research, Kansas City, MO), a plugin of ImageJ, was used to correct for XY drift. Circular regions of diameter 10 pixels were drawn to define individual bleached spines as well as non-bleached control spines, and integrated values were obtained at all time points. Values were background subtracted. Baseline fluorescence intensity was normalized to 1 . FRAP was calculated as the fluorescence increase between time 0 immediately after photobleaching and the indicated time points. Additionally, randomly selected spines in each field that were not bleached were quantified at each time point as controls for bleaching that occurred during image acquisition. The bleached spines were subsequently normalized to the average intensity of the unbleached spines over time.

To measure spine enrichment of SEP-GluA1 we first measured additional ROIs on dendritic regions immediately near each individual spine and offset from areas with hot spots of SEP-GluA1 so as to avoid other synapses. Spine enrichment was defined as the following ratio of background subtracted initial fluorescence intensity: $\left(S E P_{\text {spine }} / D s R e d_{\text {spine }}\right) /\left(S E P_{\text {dendrite }} / D s R e d_{\text {dendrite }}\right)$.

Spine intensity was quantified as DsRed cell fill fluorescence intensity within ROls in the baseline image for each time series. This was used as a measure of spine size as it has been previously shown to be closely correlated (Holtmaat et al., 2005). Similarly, dendritic intensity was quantified as DsRed cell fill fluorescence intensity within dendritic shaft ROIs and used as a measure of dendritic size. The nearest neighbor distance (NND) was calculated as the shortest distance between all synapses as defined by SEP-GluA1 clusters. These clusters were automatically identified by local fluorescence intensity maxima in the baseline image for each time series so as to avoid bias in identifying synapses. NND was used as a measure of synaptic density whereby shorter NNDs reflects higher density.

\section{Statistical analysis}

Statistical analyses and graphing were performed in Prism 8 (GraphPad software). Where means are shown, errors represent SEM. Box-and-whisker blots represent the median, interquartile range and $5 \%$ and $95 \%$ of the distribution. Column scatter plots show all individual values with a horizontal line representing the median value. Correlations were fit with a linear regression and assessed for significance using Pearson correlation. Curve fitting of fluorescence recovery was performed using nonlinear regression to fit an exponential association curve defined by $Y=Y M-(Y M-Y 0)^{*} \exp \left(-k^{*} x\right)$, where $Y M$ is the maximum fluorescence, $Y O$ is starting fluorescence, $k$ is the rate constant of recovery $\left(\mathrm{min}^{-1}\right)$, and $\mathrm{x}$ is time in minutes. Outlier removal was performed using the ROUT method with false detection rate $Q=5 \%$. Where curve fits are presented the solid line represents best fit curve and shaded areas represent the $95 \%$ confidence interval of the best fit. Pairwise statistical tests were performed using unpaired $t$ tests. Comparisons of greater than two conditions were performed using one-way ANOVA. FRAP experiments were compared using multifactorial ANOVA with variables of (1) time across the experiment and (2) experimental conditions. Reported significance on graphs represent whether the interaction of these variables was statistically significant. For statistically significant results, post hoc pairwise comparisons were performed using the Sidak's multiple comparison test. Error for all statistical analyses was set at $\alpha=0.05$. Statistical significance was considered at $p<0.05$. All statistics tables are included in extended data figures.

\section{Results}

We sparsely transfected neurons by in utero electroporation of super-ecliptic pHluorin tagged GluA1 (SEPGluA1), myc-GluA2, and DsRed, which allowed for simultaneous imaging of GluA1-containing AMPARs as well as neuronal morphology. Subsequently, cranial windows were placed for two-photon imaging over the respective cortical regions as previously described and characterized (Zhang et al., 2015; Roth et al., 2020; Tan et al., 2020).

Individual spines were targeted for photobleaching with neighboring spines as unbleached control spines (Fig. $1 A$, $B)$. Fluorescence recovery was monitored to $32 \mathrm{~min}$ postbleaching for all experiments and remained stable with no significant additional recovery observed up to $42 \mathrm{~min}$ postbleaching in a subset of samples (Fig. 1C; Extended Data Fig. $1-1 A-C$ ). Comparison of mobile fractions was subsequently performed using the asymptote (YM) calculated by fit with an exponential association curve (Fig. 1C, $D$; Extended Data Figs. 1-3, 1-4). DsRed showed fluorescence recovery to near baseline, reflective of its entirely mobile constituency facilitating exchange. In contrast, SEP-GluA1 recovered to only $\sim 50 \%$ of baseline (Fig. $1 C$, $D$ ). These findings are most consistent with previous data obtained in dissociated cell culture and suggest that a significant portion of GluA1-containing AMPARs in vivo at basal states are contained within an immobile synaptic fraction. They are thus not readily interchanged likely as a result of diffusion restriction via protein interactions and molecular crowding within the postsynaptic architecture.

Consistent with previous data (Zhang et al., 2015), the initial fluorescence intensity of DsRed, reflective of spine size, strongly correlated with initial intensity of SEPGluA1, reflective of spine AMPAR content (Fig. 1E). Interestingly, the relative concentration of SEP-GluA1 in spines relative to dendrites, defined as SEP-GluA1 spine enrichment, did not correlate with the magnitude of SEPGluA1 fluorescence recovery (Fig. 1F), suggesting that the mobile fraction of GluA1-containing AMPARs is independent of the spine GluA1 level, also consistent with previous findings (Li et al., 2016). We found that the mobile fraction was also not correlated with spine DsRed intensity, an estimate of spine size; dendritic shaft DsRed intensity, an estimate of dendritic size; or NND of synapses, an estimate of synapse density (Extended Data Fig. 1-2). 

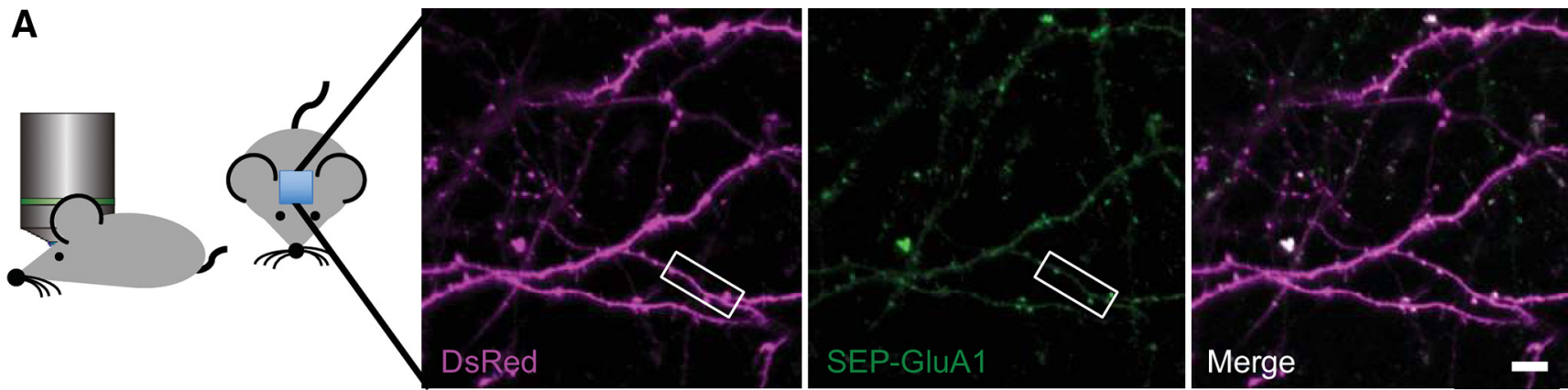

B Baseline

Bleach

FRAP

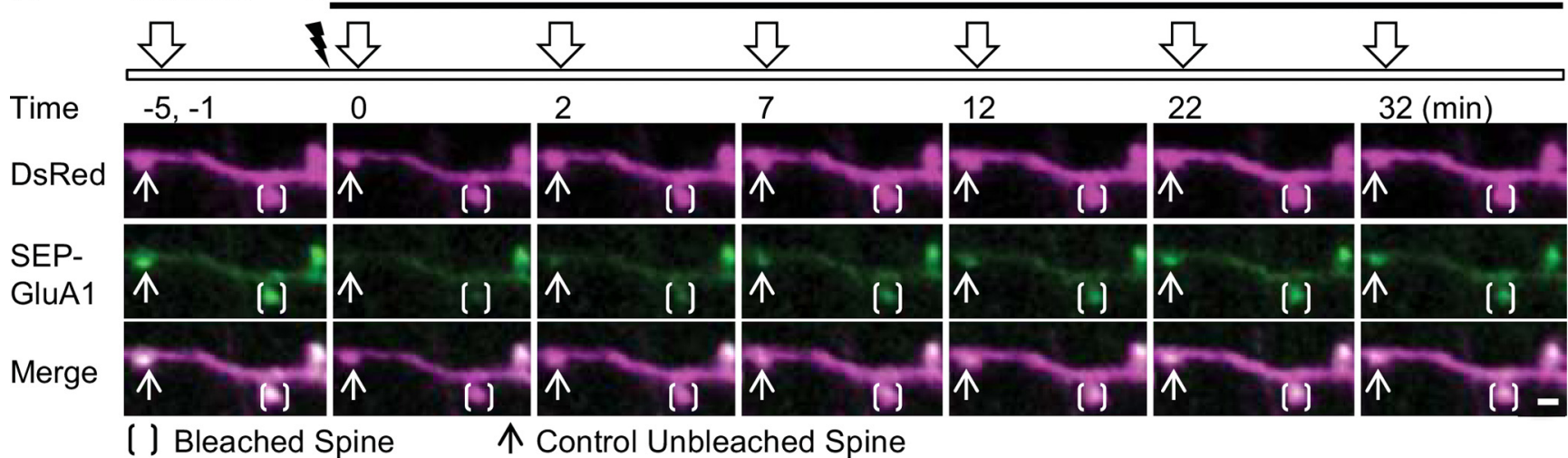

C

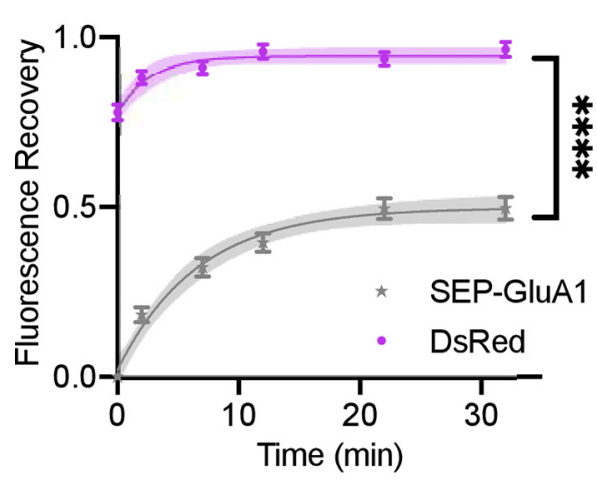

D

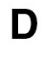

****

$\mathbf{E}$
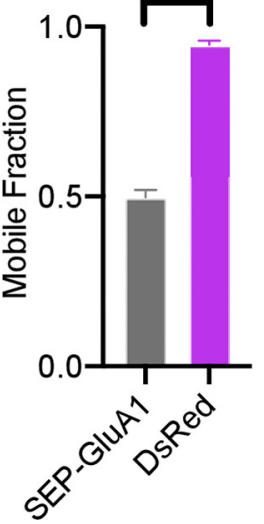

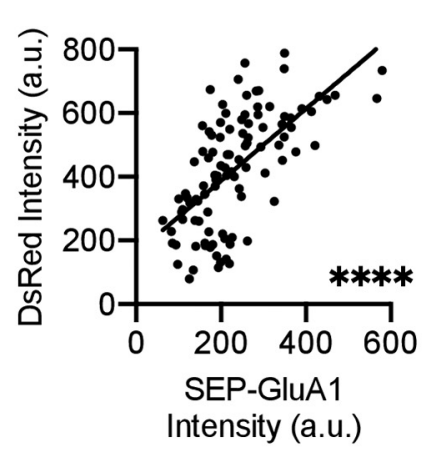

Figure 1. In vivo FRAP shows mobile and immobile fractions of GluA1 in cortical neurons. $\boldsymbol{A}$, Schematic of experimental approach using two-photon imaging of cranial windows in live mice. Representative images of maximum intensity projection (MIP) of 3D z-stack of L5 visual cortex neurons expressing DsRed cell fill (magenta), SEP-GluA1 (green), and myc-GluA2. Scale bar: $10 \mu \mathrm{m}$. Area of interest indicated corresponding to panel $\boldsymbol{B}$. B. Representative MIP image of dendrite with a bleached (bracket) and an unbleached (arrow) spine at indicated time points. Scale bar: $2 \mu \mathrm{m}$. C. Fluorescence recovery of SEP-GluA1 versus DsRed cell fill in spines (multifactorial ANOVA; Extended Data Figures 1-3, 1-4). Time points were fitted with an exponential curve indicated by solid line with $95 \% \mathrm{Cl}$ in shaded area. $\boldsymbol{D}$, Mobile fraction defined by maximum of fluorescence recovery calculated as Ymax of fitted exponential curve displayed in panel $\boldsymbol{C}$ ( $t$ test). $\boldsymbol{E}$, Correlation between initial raw intensity of DsRed cell fill and SEP-GluA1 per spine, Pearson's correlation $r^{2}=0.41$. $\boldsymbol{F}$, Correlation between recovered fraction measured at $32 \mathrm{~min}$ postbleach and spine enrichment, Pearson's correlation $r^{2}=$ 0.0006. $\boldsymbol{C}-\boldsymbol{F}, n=104$ spines, 4 mice, error bars indicate SEM; ${ }^{* \star \star} p<0.0001$, ns = not significant. See also Extended Data Figures 1-1, $1-2$.

Additionally, we sought to assess whether any differences existed between cortical areas responsible for sensory input, specifically the visual cortex, compared with motor output in the motor cortex. Thus, we compared AMPAR dynamics between neurons in L5 of motor and visual cortices (Fig. 2A,B). Furthermore, as different lamina within the cortical columnar stack possess unique inputs and feedback connections as well as distinct morphologic and electrophysiological properties (Thomson and Lamy, 2007; Gouwens et al., 2019), we also investigated whether AMPAR mobility differed within different cortical layers through comparison of neurons in L5 versus L2/3 of visual cortex (Fig. $2 C, D$ ). Overall, the total mobile fraction and spine enrichment of SEP-GluA1 in different 


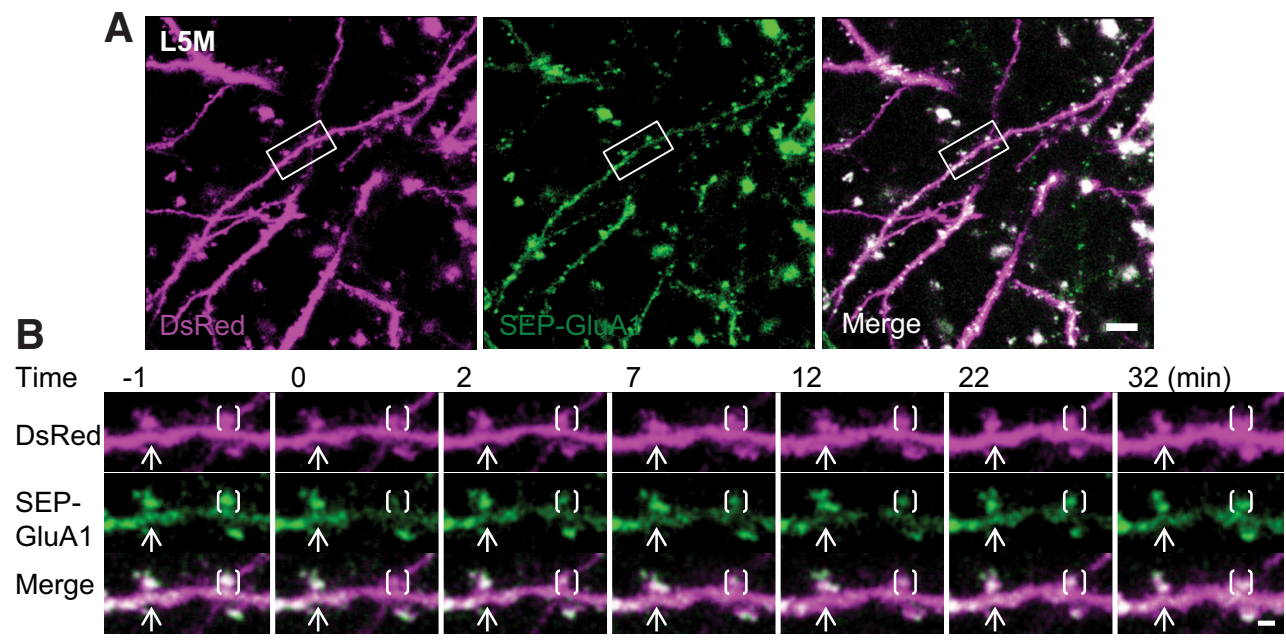

( ) Bleached Spine $\quad \uparrow$ Unbleached Control Spine

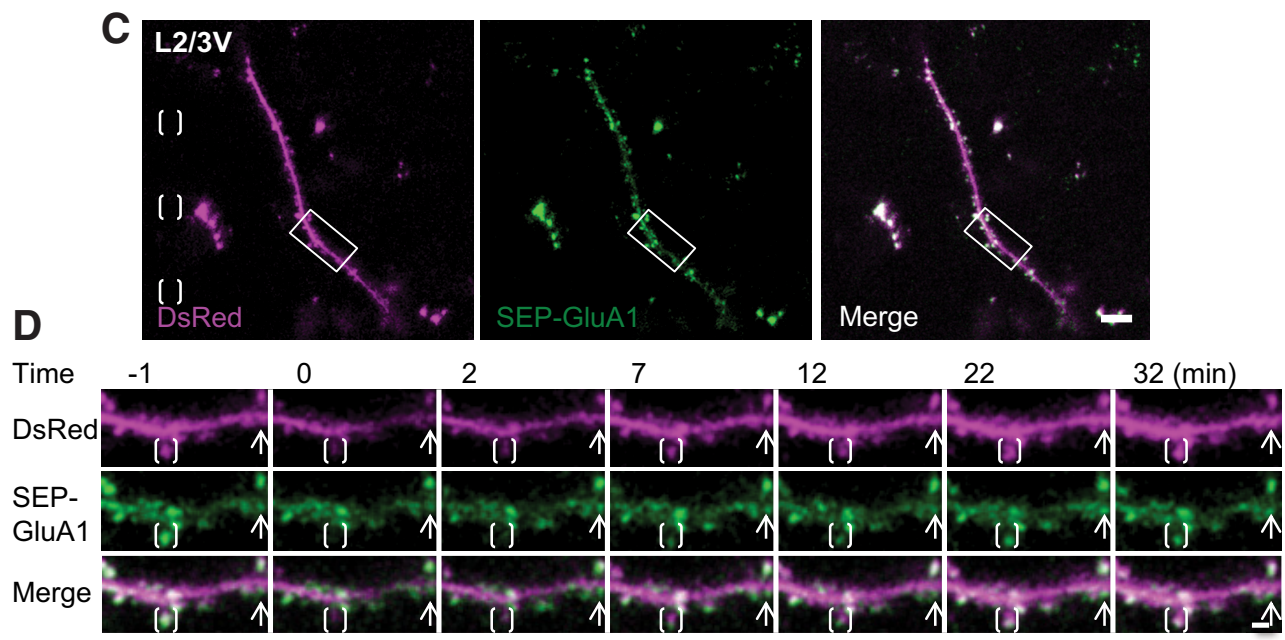

() Bleached Spine $\quad \uparrow$ Unbleached Control Spine

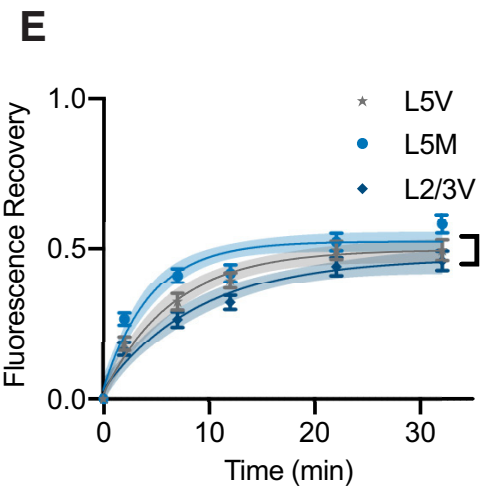

$\mathbf{F}$

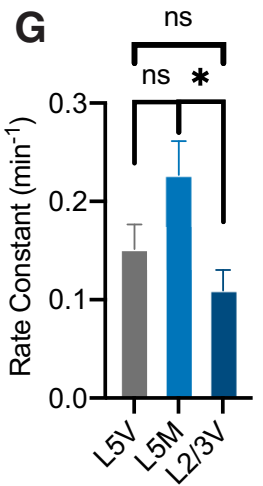

$\mathrm{H}$
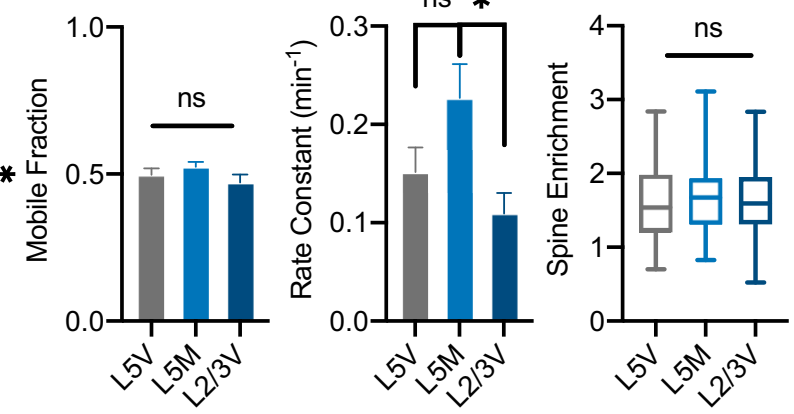

Figure 2. Similar SEP-GluA1 mobility between different cortical regions or layers. A, Representative MIP image of region of L5 motor cortex neurons expressing DsRed cell fill (magenta), SEP-GluA1 (green), and myc-GluA2. Scale bar: $10 \mu \mathrm{m}$. Area of interest indicated corresponding to panel $\boldsymbol{B}$. $\boldsymbol{B}$, Representative MIP image of bleached spine and fluorescence recovery. Scale bar: $2 \mu \mathrm{m}$. $\boldsymbol{C}, \boldsymbol{D}$, Similar to $\boldsymbol{A}, \boldsymbol{B}$ in $\mathrm{L} 2 / 3$ of visual cortex neurons (scale bars: 10 and $2 \mu \mathrm{m}$, respectively). $\boldsymbol{E}$, Fluorescence recovery of SEP-GluA1 in spines of L5 visual (L5V), L5 motor (L5M), and L2/3 visual (L2/3V) cortices (multifactorial ANOVA; Extended Data Figures 2-3, 2-4). Time points were fitted with an exponential curve indicated by solid line with $95 \% \mathrm{Cl}$ in shaded area. $\boldsymbol{F}$, Comparison of SEP-GluA1 mobile fraction across regions/layers defined by maximum fluorescence recovery calculated as Ymax of fitted exponential curves in panel $\boldsymbol{E}$ (one-way ANOVA, $p=0.24$; Extended Data Figure 2-5). G, Comparison of SEP-GluA1 recovery rate constant across regions/layers defined by k of fitted exponential curves in panel $\boldsymbol{E}$ (one-way ANOVA, Sidak's multiple comparison tests; Extended Data Figure 2-6). $\boldsymbol{H}$, Comparison of SEP-GluA1 spine enrichment across regions/layers (one-way ANOVA, $p=0.40$; Extended Data Figure 2-7). $\boldsymbol{E}-\boldsymbol{H}$, L5V $n=104$ spines, 4 mice; L5M $n=97$ spines, 3 mice; L2/3V $n=84$ spines, 3 mice, ${ }^{*} p<0.05$, ns = not significant. See also Extended Data Figures 2-1, 2-2, 2-8, 2-9, 2-10, 2-11, 2-12, 2-13. 

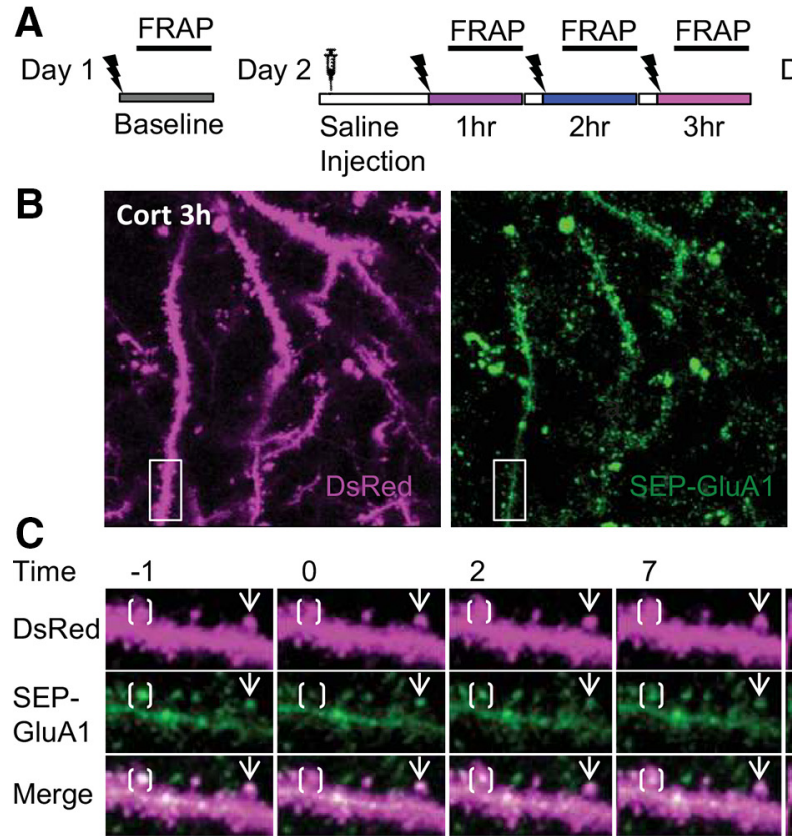

(] Bleached Spine

$\uparrow$ Unbleached Control Spine
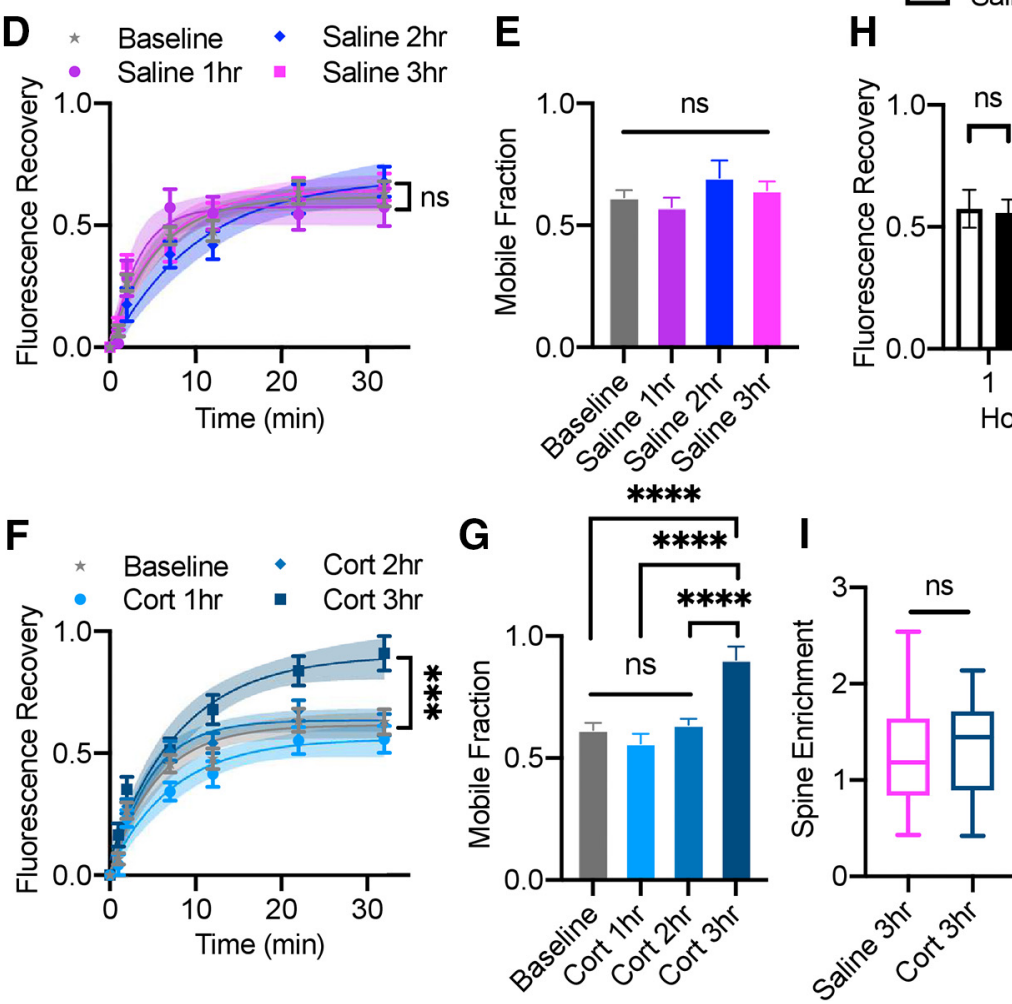

Figure 3 Corticosterone increases GluA1 mobility within spines. $\boldsymbol{A}$, Schematic of experimental design: FRAP performed on same cohort of mice across three separate days to obtain baseline and measurements at 1,2 , and $3 \mathrm{~h}$ postinjection of saline and corticosterone. B, Representative MIP image of L2/3 visual cortex neurons expressing DsRed cell fill (magenta), SEP-GluA1 (green), and myc-GluA2 $3 \mathrm{~h}$ postinjection of saline. Scale bar: $10 \mu \mathrm{m}$. Area of interest indicated corresponding to panel $\boldsymbol{C}$. $\boldsymbol{C}$, Representative MIP image of bleached (bracket) and unbleached (arrow) spines and fluorescence recovery. Scale bar: $2 \mu \mathrm{m}$. See also Extended Data Figure 3-1. $\boldsymbol{D}$, Fluorescence recovery of SEP-GluA1 in spines at baseline ( $n=106$ spines) and at $1 \mathrm{~h}(n=45 \mathrm{spines}), 2 \mathrm{~h}(n=51$ spines), and $3 \mathrm{~h}$ ( $n=53$ spines) postinjection of saline (multifactorial ANOVA; Extended Data Figures 3-3, 3-4). Time points were fitted with an exponential curve indicated by solid line with $95 \% \mathrm{Cl}$ in shaded area. $\boldsymbol{E}$, Comparison of mobile fraction at baseline and indicated times postinjection of saline defined by maximum fluorescence recovery calculated as Ymax of fitted exponential curves displayed in panel $\boldsymbol{D}$ (one-way ANOVA; Extended Data Figure 3-5). $\boldsymbol{F}$, G, Similar to $\boldsymbol{D}, \boldsymbol{E}$ at baseline and $1 \mathrm{~h}(n=43 \mathrm{spines}), 2 \mathrm{~h}$ 
continued

( $n=55$ spines), and $3 \mathrm{~h}$ ( $n=51$ spines) postinjection of corticosterone [Cort; multifactorial ANOVA for $\boldsymbol{F}$ (Extended Data Figure 3-6), one-way ANOVA with Sidak's multiple comparison tests for $\boldsymbol{G}$ (Extended Data Figure 3-7)]. $\boldsymbol{H}$, Comparison of fluorescence recovery at times postinjection of corticosterone versus saline (two-way ANOVA with Sidak's multiple comparison test; Extended Data Figure $3-8)$. $\boldsymbol{I}$, Comparison of spine enrichment at $3 \mathrm{~h}$ postinjection ( $t$ test, $p=0.43$ ). $\boldsymbol{J}$, Comparison of spine DsRed intensity normalized to nearby dendritic shaft DsRed intensity at $3 \mathrm{~h}$ postinjection ( $t$ test, $p=0.98$ ). $\boldsymbol{D}-\boldsymbol{J}, n=5$ mice, error bars indicate SEM, ${ }^{* * \star *} p<0.0001$, ${ }^{* \star *} p<0.001,{ }^{*} p<0.05$, ns = not significant. See also Extended Data Figure 3-2.

regions and layers were comparable (Fig. 2E,F,H; Extended Data Figs. 2-3, 2-4, 2-5, 2-7). However, we did observe a significant difference in the time courses of SEP-GluA1 FRAP curves with a faster rate constant of recovery within L5 of motor cortex compared with L2/3 of visual cortex (Fig. 2E,G; Extended Data Fig. 2-6). In parallel, we found that spine DsRed intensity, an estimate of spine size, varied across the regions and layers in a similar pattern as recovery rate (Extended Data Figs. $2-1 A, 2-8)$. To further assess this relationship, we pooled data from all spines across regions and layers and divided the spines into three equal bins to compare small, medium, and large spines. Indeed, we found that spine size was inversely related to recovery rate such that larger spines recovered faster than smaller spines (Extended Data Figs. 2-1D,E, 2-11, 2-12, 2-13). Meanwhile, measures of dendritic size and synaptic density did show notable differences between the different regions and layers, consistent with some prior observations (Konur et al., 2003; Holtmaat et al., 2005), but these differences did not parallel those observed in recovery rate (Extended Data Figs. 2-1B,C, 2-9, 2-10). No differences were seen between groups for FRAP of DsRed cell fill (Extended Data Fig. 2-2).

Finally, the mobility and trafficking of GluA1 within synapses have been shown to be regulated by various mechanisms, including by glucocorticoids. Specifically, in rodents, corticosterone, the main glucocorticoid, has been found to have effects on synaptic transmission and plasticity, which may underlie the influence of stress on learning and memory (Krugers et al., 2010; Timmermans et al., 2013; McEwen et al., 2015). Past studies have found that intraperitoneal injection of corticosterone increases spine formation and elimination in cortical neurons within hours of injection (Liston and Gan, 2011). Moreover, timing of corticosterone injections relative to innate circadian corticosterone peaks and troughs affects learning (Liston et al., 2013). Furthermore, application of corticosterone to cultured hippocampal neurons increased both GluA1-containing and GluA2-containing AMPAR surface mobility and surface expression (Heine et al., 2008; Martin et al., 2009). Particularly pronounced effects on AMPAR mobility were seen $3 \mathrm{~h}$ following corticosterone application whereby FRAP imaging revealed complete FRAP, suggesting complete loss of the immobile pool of synaptic AMPARs following treatment (Martin et al., 2009).

We therefore sought to determine whether corticosterone would have similar effects on AMPAR mobility in synapses in vivo. We performed repeated measures comparisons of AMPAR mobility within different subsets of dendritic spines of the same mice intraperitoneally injected with corticosterone (dissolved in $0.9 \%$ normal saline) versus normal saline on different days up to $3 \mathrm{~h}$ postinjection compared with baseline with no injection (Fig. 3; Extended Data Fig. 3-1). We found that SEP-GluA1 fluorescence recovery significantly increased to $\sim 90 \% 3 \mathrm{~h}$ following injection of corticosterone compared with similar fluorescence recovery levels between baseline and 1 to $2 \mathrm{~h}$ following injection (Fig. 3F,G; Extended Data Figs. 3-6, 37). In contrast, injection of saline produced no such change (Fig. 3D,E; Extended Data Figs. 3-3, 3-4, 3-5). Comparison of fluorescence recovery at $32 \mathrm{~min}$ after photobleaching showed significantly higher recovery of SEP-GluA1 $3 \mathrm{~h}$ following corticosterone injection compared with $3 \mathrm{~h}$ following saline injection (Fig. $3 H$; Extended Data Fig. 3-8). This suggests that corticosterone injection causes a shift from a near equal split between mobile and immobile pools of GluA1-containing receptors to an almost entirely mobile pool of GluA1-containing receptors within cortical synapses, which is consistent with that previously seen within dissociated hippocampal cultures (Groc et al., 2008; Martin et al., 2009). This observed difference in receptor mobility was not associated with differences between SEP-GluA1 spine enrichment or spine DsRed intensity, suggesting corticosterone induced increase in mobile fraction was unlikely because of change in receptor spine enrichment or spine size (Fig. $3 /, J)$. No differences were observed in FRAP of DsRed cell fill FRAP (Extended Data Fig. 3-2).

\section{Discussion}

This study shows that within cortical synapses in vivo, there exist populations of mobile and immobile GluA1-containing receptors in basal states. This is generally consistent with previous findings in dissociated cell culture studies (Ashby et al., 2006; Bats et al., 2007; Heine et al., 2008; Frischknecht et al., 2009; Martin et al., 2009; Arendt et al., 2010). On the other hand, this is incongruent with findings in organotypic slice cultures that suggest GluA1containing receptors are entirely mobile in basal states and are only incorporated into immobile synaptic fractions during LTP (Hayashi et al., 2000; Kopec et al., 2006; Makino and Malinow, 2009). Additionally, the existence of an immobile fraction of GluA1-containing receptors suggests that these receptors are present within synapses and do not solely reside within spine surface receptors pools. This would also suggest that the presence of the SEP tag on recombinant GluA1-containing receptors does not intrinsically interfere with its trafficking to synapses as has been suggested (Díaz-Alonso et al., 2017). These differences likely result from the different preparations and experimental conditions, highlighting the need for experimentation within more intact systems.

We further observe that the proportion of GluA1-containing receptors is similar across different cortical areas of the 
brain as well as different layers within the visual cortex. Our data suggest that across the different regions and layers, larger spine sizes may be associated with higher rates of receptor mobility. This finding is consistent with previous work showing that spine morphology affects diffusion of molecules within spines, and more specifically, that spine volume positively correlates with FRAP recovery rate (Tønnesen et al., 2014). As such, spine morphology is proposed to confer compartmentalized signaling capacity to synapses allowing synaptic strength to be regulated independently from neighboring synapses. Moreover, we find that the proportion of mobile to immobile GluA1-containing receptors may be altered by corticosterone in a time-dependent manner. This suggests that AMPAR mobility is a fluid parameter within cortical neurons that may be regulated by stress to modulate basal synaptic strength or synaptic plasticity. Overall, these findings point to common basic properties of glutamatergic receptors that likely reflect their tight regulation within synapses given their critical function within the nervous system.

\section{References}

Arendt KL, Royo M, Fernández-Monreal M, Knafo S, Petrok CN, Martens JR, Esteban JA (2010) PIP 3 controls synaptic function by maintaining AMPA receptor clustering at the postsynaptic membrane. Nat Neurosci 13:36.

Ashby MC, Maier SR, Nishimune A, Henley JM (2006) Lateral diffusion drives constitutive exchange of AMPA receptors at dendritic spines and is regulated by spine morphology. J Neurosci 26:7046-7055.

Bats C, Groc L, Choquet D (2007) The interaction between Stargazin and PSD-95 regulates AMPA receptor surface trafficking. Neuron 53:719-734.

Borgdorff AJ, Choquet D (2002) Regulation of AMPA receptor lateral movements. Nature 417:649-653.

Díaz-Alonso J, Sun YJ, Granger AJ, Levy JM, Blankenship SM, Nicoll RA (2017) Subunit-specific role for the amino-terminal domain of AMPA receptors in synaptic targeting. Proc Natl Acad Sci USA 114:7136-7141.

Diering GH, Huganir RL (2018) The AMPA receptor code of synaptic plasticity. Neuron 100:314-329.

Frischknecht R, Heine M, Perrais D, Seidenbecher Cl, Choquet D, Gundelfinger ED (2009) Brain extracellular matrix affects AMPA receptor lateral mobility and short-term synaptic plasticity. Nat Neurosci 12:897-904.

Gouwens NW, Sorensen SA, Berg J, Lee C, Jarsky T, Ting J, Sunkin SM, Feng D, Anastassiou CA, Barkan E (2019) Classification of electrophysiological and morphological neuron types in the mouse visual cortex. Nat Neurosci 22:1182-1195.

Groc L, Choquet D, Chaouloff F (2008) The stress hormone corticosterone conditions AMPAR surface trafficking and synaptic potentiation. Nat Neurosci 11:868.

Hayashi Y, Shi S-H, Esteban JA, Piccini A, Poncer J-C, Malinow R (2000) Driving AMPA receptors into synapses by LTP and CaMKII: requirement for GluR1 and PDZ domain interaction. Science 287:2262-2267.

Heine M, Groc L, Frischknecht R, Béique JC, Lounis B, Rumbaugh G, Huganir RL, Cognet L, Choquet D (2008) Surface mobility of postsynaptic AMPARs tunes synaptic transmission. Science 320:201-205.

Holtmaat AJ, Trachtenberg JT, Wilbrecht L, Shepherd GM, Zhang X, Knott GW, Svoboda K (2005) Transient and persistent dendritic spines in the neocortex in vivo. Neuron 45:279-291.
Huganir R, Nicoll R (2013) AMPARs and synaptic plasticity: the last 25 years. Neuron 80:704-717.

Konur S, Rabinowitz D, Fenstermaker VL, Yuste R (2003) Systematic regulation of spine sizes and densities in pyramidal neurons. $J$ Neurobiol 56:95-112.

Kopec CD, Li B, Wei W, Boehm J, Malinow R (2006) Glutamate receptor exocytosis and spine enlargement during chemically induced long-term potentiation. J Neurosci 26:2000-2009.

Krugers HJ, Hoogenraad CC, Groc L (2010) Stress hormones and AMPA receptor trafficking in synaptic plasticity and memory. Nat Rev Neurosci 11:675-681.

Li TP, Song Y, MacGillavry HD, Blanpied TA, Raghavachari S (2016) Protein crowding within the postsynaptic density can impede the escape of membrane proteins. J Neurosci 36:4276-4295.

Liston C, Gan WB (2011) Glucocorticoids are critical regulators of dendritic spine development and plasticity in vivo. Proc Natl Acad Sci USA 108:16074-16079.

Liston C, Cichon JM, Jeanneteau F, Jia Z, Chao MV, Gan WB (2013) Circadian glucocorticoid oscillations promote learning-dependent synapse formation and maintenance. Nat Neurosci 16:698.

MacGillavry HD, Song Y, Raghavachari S, Blanpied TA (2013) Nanoscale scaffolding domains within the postsynaptic density concentrate synaptic AMPA receptors. Neuron 78:615-622.

Makino H, Malinow R (2009) AMPA receptor incorporation into synapses during LTP: the role of lateral movement and exocytosis. Neuron 64:381-390.

Martin S, Henley JM, Holman D, Zhou M, Wiegert O, Van Spronsen M, Joëls M, Hoogenraad CC, Krugers HJ (2009) Corticosterone alters AMPAR mobility and facilitates bidirectional synaptic plasticity. PLoS One 4:e4714.

McEwen BS, Bowles NP, Gray JD, Hill MN, Hunter RG, Karatsoreos IN, Nasca C (2015) Mechanisms of stress in the brain. Nat Neurosci 18:1353.

Nair D, Hosy E, Petersen JD, Constals A, Giannone G, Choquet D, Sibarita J-B (2013) Super-resolution imaging reveals that AMPA receptors inside synapses are dynamically organized in nanodomains regulated by PSD95. J Neurosci 33:13204-13224.

Roth RH, Cudmore RH, Tan HL, Hong I, Zhang Y, Huganir RL (2020) Cortical synaptic AMPA receptor plasticity during motor learning. Neuron 105:895-908.e5.

Sharma K, Fong DK, Craig AM (2006) Postsynaptic protein mobility in dendritic spines: long-term regulation by synaptic NMDA receptor activation. Mol Cell Neurosci 31:702-712.

Tan HL, Roth RH, Graves AR, Cudmore RH, Huganir RL (2020) Lamina-specific AMPA receptor dynamics following visual deprivation in vivo. Elife 9:e52420.

Tang AH, Chen H, Li TP, Metzbower SR, MacGillavry HD, Blanpied TA (2016) A trans-synaptic nanocolumn aligns neurotransmitter release to receptors. Nature 536:210-214.

Thomson AM, Lamy C (2007) Functional maps of neocortical local circuitry. Front Neurosci 1:19-42.

Timmermans W, Xiong H, Hoogenraad C, Krugers H (2013) Stress and excitatory synapses: from health to disease. Neuroscience 248:626-636.

Tønnesen J, Katona G, Rózsa B, Nägerl UV (2014) Spine neck plasticity regulates compartmentalization of synapses. Nat Neurosci 17:678-685.

Wakayama S, Kiyonaka S, Arai I, Kakegawa W, Matsuda S, Ibata K, Nemoto YL, Kusumi A, Yuzaki M, Hamachi I (2017) Chemical labelling for visualizing native AMPA receptors in live neurons. Nat Commun 8:14850.

Zhang Y, Cudmore RH, Lin D-T, Linden DJ, Huganir RL (2015) Visualization of NMDA receptor-dependent AMPA receptor synaptic plasticity in vivo. Nat Neurosci 18:402-407. 